\title{
do it yourself
}

\author{
Vivienne Baillie Gerritsen
}

It can take ages to meet the right partner. So much so that plants lost their patience millions of years ago and thought up something else: the art of selfing. Many flowering plants are indeed capable of extensive in-breeding - by way of a rather subtle form of hermaphroditism - to ensure their spread and survival. The common mouse-ear cress, Arabidopsis thaliana, which has become the model plant for botanists, is revealing how many plants are able to perpetuate their species by letting their pollen fertilise their own pistil. Which prompts the question: how does any given plant species avoid self-fertilisation in the first place? The answer, or at least part of it, is: the $S$ locus. The S locus carries two genes whose protein products - SCR and SRK - are directly involved in A.thaliana's capacity to self-pollinate or not, and may well illustrate the pathway used by many other plants.

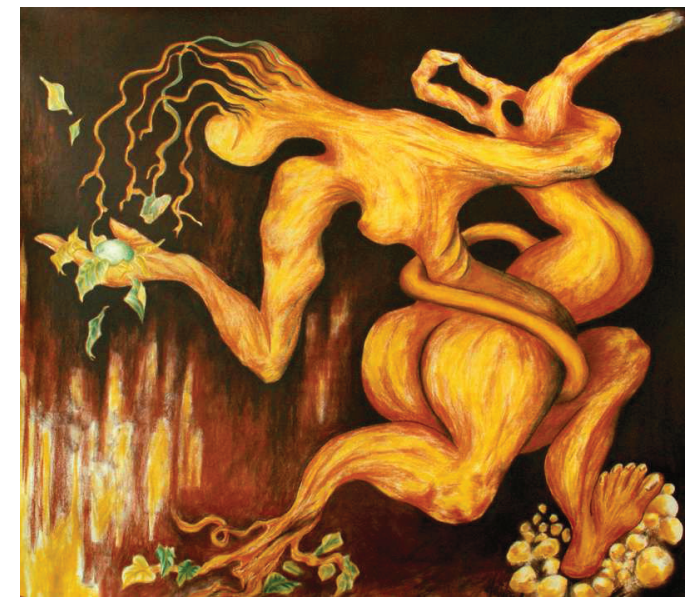

"Hermafrodite", by Lisbeth Hummel

Courtesy of the artist

The notion that plants are able to self-pollinate is not new. Charles Darwin, who is widely known for his thoughts on the evolution of primates, also spent a lot of time making observations on countless other organisms, including plants. He had already suggested the existence of self-fertilisation during the second half of the $19^{\text {th }}$ century - and even dedicated a book to the notion, The Effects of Cross and Self Fertilisation in the Vegetable Kingdom (1876).
Why would plants seek to self-pollinate? The answer seems obvious. When you can't find someone else to do it, do it yourself. It is not in the pursuit of pleasure that a plant would end up self-pollinating but rather in the hope of guaranteeing successful growth within a given environment; an environment that has become hostile enough to make cross-breeding difficult, yet in which the disadvantages of in-breeding are outweighed by the advantages...

In the past few years, scientists have been studying one particular system in Arabidopsis thaliana, which has proved to be an essential part of an elegant process that has been coined "self-incompatibility" (SI). SI is used by plants to avoid self-pollination, thus ensuring genetic variation and population vigour. In a nutshell, plants whose SI system is working properly are not able to self-pollinate. It sounds straightforward enough, yet the SI system is turning out to be a complex one. However, what has been termed the $\mathrm{S}$ locus seems to play a pivotal role and is best illustrated by its protein products: s-locus receptor kinase (SRK) and slocus cysteine-rich protein (SCR).

SRK is a transmembrane receptor protein, which probably forms a homodimer and is found on the very tip of a flower's pistil, known as its stigma. The stigma forms a kind of platform on which pollen is able to land, hydrate, germinate, and ultimately push its pollen tube all the way down the pistil to the 
ovary to complete fertilisation. SCR is found in the pollen's outer coat and is secreted when pollen approaches the stigma. If the pollen and the pistil belong to the same plant, SCR and SRK belong to the same S locus and, like glove in hand, SCR will bind to SRK. Their binding then triggers off an alert system - probably via SRK phosphorylation - which interrupts pollen tube growth. As such, the system functions much like passing through customs - if the pollen belongs to the same plant, an alarm goes off and fertilisation is immediately stopped. It has been suggested that this happens by the degradation of actin filaments which support pollen tube growth.

This is how SI works for many plants. In A.thaliana, however, due to numerous mutations over time, the S locus has been out of order for about half a million years. As a consequence, when A.thaliana's pollen settles on the tip of its own pistils, SCR is not recognised by the plant's SRK. Consequently, the SI alert is not set off and the pollen is left to germinate and make its way down the pistil to the ovary.

As always, no given pathway can be trimmed down to the likes of one or two proteins. Indeed, though a key element in plant selfincompatibility, the $\mathrm{S}$ locus is not the only decision-making entity in the SI pathway. A cascade of decision events occurs downstream and many other processes - such as pollen/stigma recognition, pollen hydration, germination and directional growth - upstream. Nevertheless, A.thaliana is turning out to be an excellent plant model for studying signalling pathways.

From a purely biological point of view, inbreeding - not to mention incest - has never been encouraged within a species, mainly for its healthy survival. Here's a thought: is it not amazing that the plant kingdom has resorted to a genetic system to discourage in-breeding, while humans count on words and cultural heritage?

\section{Cross-references to UniProt}

Defensin-like protein A, SCRA, Arabidopsis thaliana (Mouse-ear cress) : P0CG07

S-receptor-like serine/threonine-protein kinase SRK, Arabidopsis thaliana (Mouse-ear cress) : B0F2A9

\section{References}

1. Ivanov R., Fobis-Loisy I., Gaude T.

When no means no: guide to Brassicaceae self-incompatibility

Trends in Plant Science 15:387-394(2010)

PMID: 20621670

2. Tsuchimatsu T., Suwabe K., Shimizu-Inatsugi R., Isokawa S., Pavlidis P., Staedler T., Suzki G., Takayama S., Watanabe M., Shimizu K.K.

Evolution of self-compatibility in Arabidopsis by a mutation in the male specificity gene

Nature 464:1342-1347(2010)

PMID: 20400945

3. Boggs N.A., Nasrallah J.B., Nasrallah M.E.

Independent S-locus mutations caused self-fertility in Arabidopsis thaliana

PLoS Genetics. Volume 5, issue 3

PMID: 19300485

Protein Spotlight (ISSN 1424-4721), http://www.proteinspotlight.org, is published by the Swiss-Prot group at the Swiss Institute of Bioinformatics (SIB). Authorization to photocopy or reproduce this article for internal or personal use is granted by the SIB provided its content is not modified. Please enquire at spotlight@isb-sib.ch for redistribution or commercial usage. 